\title{
Role of IL-1 $\alpha$ and the NIrp3/caspase-1/L-1 $\beta$ axis in cigarette smoke-induced pulmonary inflammation and COPD
}

\author{
N.S. Pauwels*, K.R. Bracke*, L.L. Dupont*, G.R. Van Pottelberge*, S. Provoost*, \\ T. Vanden Berghe ${ }^{\#, \oplus}$, P. Vandenabeele ${ }^{\#, \oplus, ~ B . N . ~ L a m b r e c h t ~}{ }^{+}$, \\ G.F. Joos* and G.G. Brusselle*
}

ABSTRACT: Cigarette smoke (CS), the primary risk factor of chronic obstructive pulmonary disease (COPD), leads to pulmonary inflammation through interleukin-1 receptor (IL-1R)I signalling, as

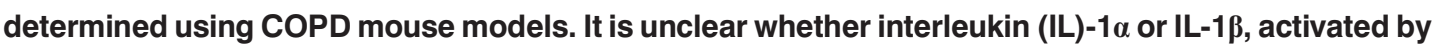
the NIrp3/caspase-1 axis, is the predominant ligand for IL-1RI in CS-induced responses.

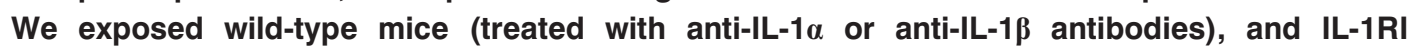
knockout (KO), NIrp3 KO and caspase-1 KO mice to CS for 3 days or 4 weeks and evaluated

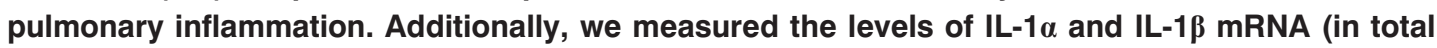
lung tissue by RT-PCR) and protein (in induced sputum by ELISA) of never-smokers, smokers without COPD and patients with COPD.

In CS-exposed mice, pulmonary inflammation was dependent on IL-1RI and could be significantly attenuated by neutralising IL-1 $\alpha$ or IL-1 $\beta$. Interestingly, CS-induced inflammation

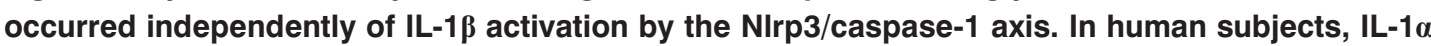
and IL-1ß were significantly increased in total lung tissue and induced sputum of patients with COPD, respectively, compared with never-smokers.

These results suggest that not only IL-1 $\beta$ but also IL-1 $\alpha$ should be considered as an important mediator in CS-induced inflammation and COPD.

KEYWORDS: Caspase-1, chronic obstructive pulmonary disease, cigarette smoke, inflammasome, interleukin-1, NIrp3

hronic obstructive pulmonary disease (COPD), a primarily cigarette smoke (CS)related disease, is a leading cause of morbidity and mortality worldwide. Its overall prevalence in adults $>40$ yrs of age is currently estimated at $10 \%$ [1]. Inflammation is present in the lungs of patients with COPD, and is critical in the development and progression of the disease, leading to obstructive bronchiolitis and destruction of lung parenchyma (emphysema) [2]. Current COPD therapy mainly focuses on the reduction of symptoms, such as cough and breathlessness, whereas effective anti-inflammatory therapies are still lacking. The aim of this study was to unravel the molecular mechanisms involved in CS-induced pulmonary inflammation, which is of vital importance for the development of future antiinflammatory therapies for patients with COPD.

Interleukin (IL)-1 $\alpha$ and IL-1 $\beta$, two pro-inflammatory isoforms of the IL- 1 family of ligands, play an important role in modulating both innate and adaptive immune responses [3, 4]. They share a common receptor, IL-1 receptor (IL-1R)I, which activates the transcription factors nuclear factor$\kappa \mathrm{B}$ and activator protein-1 upon stimulation. Eventually, the ensuing pathway increases the expression of vascular adhesion molecules (e.g. intercellular adhesion molecule- 1 and vascular cellular adhesion molecule-1) and induces chemokines (e.g. IL-8, a neutrophil chemoattractant) that together promote inflammatory cell infiltration from the circulation into the affected tissue(s) [3-5]. Importantly, IL-1 $\beta$ is produced as an inactive precursor form and its activation is generally performed by active caspase-1 (IL- $1 \beta$-converting enzyme) [3]. Pro-caspase-1 can itself be activated by the Nlrp3 (NOD-like receptor family, pyrin domain containing 3) inflammasome, a multiprotein platform activated upon nonmicrobial and stress-associated danger signals, including reactive oxygen species and extracellular adenosine triphosphate [6]. However, cleavage of procaspase-1 is not exclusively performed by the
AFFILIATIONS

*Laboratory for Translational Research in Obstructive Pulmonary Diseases,

+Laboratory of Immunoregulation and Mucosal Immunity, Dept of Respiratory Medicine, Ghent University,

\#Dept of Biomedical Molecular Biology, Ghent University, and - Dept for Molecular Biomedical Research, Flanders Institute for Biotechnology, Ghent, Belgium.

CORRESPONDENCE

G.G. Brusselle

Laboratory for Translational Research in Obstructive Pulmonary Diseases, Dept of Respiratory Medicine Ghent University Hospital

$7 \mathrm{~K} 12 \mathrm{E}$

De Pintelaan 185

B-9000 Ghent

Belgium

E-mail: Guy.Brusselle@UGent.be

Received:

Oct 082010

Accepted after revision:

April 112011

First published online:

May 262011

European Respiratory Journal Print ISSN 0903-1936 Online ISSN 1399-3003 
Nlrp3 inflammasome, as caspase- 1 can also be activated by other inflammasomes [7-9]. In contrast with IL-1 $\beta$, both the precursor and cleaved forms of IL-1 $\alpha$ are biologically active. IL$1 \alpha$ is released by dying cells, acting as a danger signal [10], but it can also be secreted by a caspase-1-dependent mechanism [11].

Animal models that mimic the hallmarks of COPD are a valuable tool in unravelling the molecular mechanisms of the inflammatory process, and have already established an important role for IL-1RI in acute and chronic CS-induced inflammation [12, 13]. Our study demonstrates for the first time that subacute (4 weeks) CS-induced inflammation is also IL-1RI dependent, which reinforces the concept that the IL-1RI pathway is critically implicated in CS-induced responses. However, it is still unclear which pro-inflammatory ligand of IL-1RI, IL- $1 \alpha$ or IL- $1 \beta$, predominantly stimulates its receptor in CS-induced responses. To elucidate this, we injected mice intravenously with antibodies against IL- $1 \alpha$ or IL-1 $1 \beta$, exposed them to CS for 3 days (acute exposure) and studied pulmonary cell accumulation. Next, we investigated the role of Nlrp3 and caspase-1, upstream regulators of IL-1 $\beta$, in prolonged CSinduced inflammation, by exposing wild-type (WT), Nlrp3 knockout (KO) and caspase-1 KO mice to CS for 4 weeks (subacute exposure). In addition, we performed a translational study investigating mRNA and protein expression of IL- $1 \alpha$ and IL-1 $\beta$ (in total lung tissue and sputum supernatants, respectively) of never-smokers, smokers without COPD and patients with COPD.

\section{MATERIALS AND METHODS \\ Animals}

Homozygous breeding pairs of IL-1RI KO mice (B6.129S7$\operatorname{Il1r} 1^{\operatorname{tm} 1 \operatorname{Imx}}$ ) and control WT mice (C57BL/6J) were obtained from the Jackson Laboratory (Bar Harbor, ME, USA). Nlrp3 KO mice (and appropriate control WT mice) were kindly provided by J. Tschopp (University of Lausanne, Lausanne, Switzerland). Caspase-1 KO mice were obtained from P. Vandenabeele (Flanders Institute for Biotechnology, Ghent, Belgium). Except for the caspase- $1 \mathrm{KO}$ and control mice, which were bred in the animal facility at the Flanders Institute for Biotechnology, all animals were bred in the animal facility at the Faculty of Medicine and Health Sciences (Ghent University, Ghent). All mice used in this study were bred on a C57BL/6J background. Animals of age 6-10 weeks were maintained in standard conditions under a $12 \mathrm{~h}$ light-dark cycle, and provided with a standard diet (Pavan, Brussels, Belgium) and chlorinated tap water ad libitum. All in vivo manipulations were approved by the local ethics committee for animal experimentation of the Faculty of Medicine and Health Sciences.

\section{CS exposure}

Groups of 8-10 mice were exposed to CS, as described previously [14]. Briefly, the animals received mainstream CS from five reference cigarettes (3R4F without filter; University of Kentucky, Lexington, KY, USA) four times a day with 30-min smoke-free intervals. An optimal smoke/air ratio of $1 / 6$ was obtained. The mice were exposed for 3 days (acute) or 4 weeks (subacute). The control groups were exposed to room air.

\section{Administration of anti-IL-1 $\alpha$ and anti-IL-1 $\beta$ antibodies}

C57BL/ 6 mice were injected i.v. with $40 \mu \mathrm{g}$ anti-IL-1 $\alpha$, anti-IL$1 \beta$ or Armenian hamster immunoglobulin (Ig)G isotype control antibodies (Biolegend, San Diego, CA, USA). Mice were injected on days 1 and 3 of an acute (3 days) CS experiment, $30 \mathrm{~min}$ before air or CS exposure.

\section{Bronchoalveolar lavage}

Bronchoalveolar lavage (BAL) was performed as previously described [15-17]. Briefly, lungs were first lavaged using $3 \times 300 \mu \mathrm{L}$ Hank's buffered salt solution (HBSS), which was free of $\mathrm{Ca}^{2+}$ and $\mathrm{Mg}^{2+}$, and supplemented with $1 \%$ bovine serum albumin (BSA), followed by $3 \times 1 \mathrm{~mL}$ HBSS supplemented with $0.6 \mathrm{mM}$ EDTA, via a tracheal cannula. The six lavage fractions were pooled, centrifuged and the cell pellet was finally resuspended in $200 \mu \mathrm{L}$ buffer (PBS supplemented with $1 \%$ BSA, $5 \mathrm{mM}$ EDTA and $0.1 \%$ sodium azide). Subsequently, total cell counts were obtained using a Bürker chamber and differential cell counts ( $\geqslant 400$ cells) were performed on cytocentrifuged preparations after May-Grünwald (Sigma-Aldrich, St Louis, MO, USA) and Giemsa staining (VWR, West Chester, PA, USA). Flow cytometric analysis of BAL cells was performed to count macrophages, neutrophils, dendritic cells (DCs), and CD4+ and CD8+ T-cells.

\section{Lung harvesting and preparation of lung single-cell suspensions}

Following BAL, the pulmonary and systemic circulation was rinsed with saline supplemented with $5 \mathrm{mM}$ EDTA. The left lung was used for histology, as previously described [5, 15]. The right lung was harvested for the preparation of lung homogenate (middle lobe) and single-cell suspension (major lobe), as described previously [18]. Briefly, the lung was thoroughly minced, digested, subjected to red blood cell lysis, passed through a $50-\mu \mathrm{m}$ cell strainer and kept on ice until labelling. Cell counting was performed with a Z2 particle counter (Beckman-Coulter Inc., Fullerton, CA, USA).

\section{Labelling of BAL cells and lung single-cell suspension for flow cytometry}

The cells were first incubated with Fc receptor-blocking antibody (anti-CD16/CD32; clone 2.4G2) to reduce nonspecific binding. Labelling reactions were then performed to discriminate macrophages, DCs and T-cells. All reactions were performed on ice. The macrophages and DCs were discriminated using the methodology described by VermaELEN and PAuweLs [19]. Briefly, macrophages are identified as a CD11c-bright (allophycocyanin (APC)-conjugated anti-CD11c; HL3), high-autofluorescence cell population. DCs were characterised as a CD11c-bright, low-autofluorescence and major histocompatibility complex class II-bright (phycoerythrin (PE)-conjugated anti-I-A[b]; AF6120.1) population. Mouse T-cell subpopulations in lung singlecell suspensions were identified using the following antibodies: fluorescein isothiocyanate (FITC)-conjugated anti-CD4 (GK1.5), FITC-conjugated anti-CD8 (53-6.7), APC-conjugated anti-CD3 (145-2C11) and PE-conjugated anti-CD69 (H1.2F3), a marker for activation of T-cells. All monoclonal antibodies were obtained from BD Pharmingen (San Diego, CA, USA). Finally, all samples were incubated with 7-amino-actinomycin D for exclusion of dead cells (BD Pharmingen).

Flow cytometry data acquisition was performed using a duallaser FACSCalibur ${ }^{\mathrm{TM}}$ flow cytometer running CellQuest ${ }^{\mathrm{TM}}$ software (BD Biosciences, San Diego, CA, USA). FlowJo 
software (Tree Star Inc., Ashland, OR, USA) was used for data analysis.

\section{Preparation of lung tissue homogenate}

The middle lobe of the right lung was snap frozen (in liquid nitrogen) and stored at $-80^{\circ} \mathrm{C}$ until further analysis. The lobes were transferred to tubes containing $1 \mathrm{~mL}$ T-PER tissue protein extraction reagent containing Halt ${ }^{\mathrm{TM}}$ Protease Inhibitor Cocktail Kit (Thermo Fisher Scientific, Waltham, MA, USA) and homogenised on ice using TissueRuptor (Qiagen, Hilden, Germany). The homogenates were centrifuged $\left(10,000 \times g\right.$ for $5 \mathrm{~min}$ at $\left.4^{\circ} \mathrm{C}\right)$ and the middle layer was transferred to microcentrifuge tubes. Total protein concentration was measured using the Bradford protein assay (Bio-Rad Laboratories, Hercules, CA, USA). Lung tissue homogenates were diluted with T-PER cocktail kit to a final protein concentration of $500 \mu \mathrm{g} \cdot \mathrm{mL}^{-1}$.

\section{IL-1 $\alpha$ and IL-1 $\beta$ ELISA}

We determined IL- $1 \alpha$ and IL- $\beta$ in BAL fluid and lung homogenate using commercially available ELISA kits (R\&D Systems, Minneapolis, MN, USA). ELISA was performed according to the manufacturer's instructions.

\section{Human study populations}

Lung resection specimens were obtained from 57 patients diagnosed with solitary pulmonary tumours at Ghent University Hospital (Ghent). None of the patients were treated with neo-adjuvant chemotherapy. Sputum induction was performed on 53 subjects who were recruited from the outpatient pulmonary clinic of Ghent University Hospital or by advertising. There was no overlap between the subjects for lung tissue and sputum analysis. All subjects were classified into three groups: never-smokers, smokers without COPD and patients with COPD. Written informed consent was obtained from all subjects according to protocols approved by the medical ethical committee of Ghent University Hospital.

\section{Lung tissue isolation, RNA extraction and real-time PCR analysis}

Lung tissue at maximum distance from the pulmonary lesions and without signs of retro-obstructive pneumonia or tumour invasion was collected by a pathologist. Tissue blocks from the resection samples were submersed in RNAlater (Ambion ${ }^{\circledR}$, Lennik, Belgium) and stored at $-80^{\circ} \mathrm{C}$ until RNA extraction.

Total lung RNA was extracted with the RNeasy Mini kit (Qiagen) and cDNA was obtained using the Transcriptor First Strand cDNA synthesis kit (Roche, Basel, Switzerland) according to the manufacturer's instructions. Expression of the target genes encoding IL-1 $\alpha$ and IL-1 $\beta$, and reference genes GAPDH (encoding glyceraldehyde-3-phosphate dehydrogenase), HPRT-1 (encoding hypoxanthine phosphoribosyltransferase-1) and PPIA (encoding peptidylprolyl isomerase 1) were analysed using TaqMan Gene Expression Assays (Applied Biosystems, Foster City, CA, USA). Real-time PCR reactions were set up in duplicate using diluted cDNA and identical amplification conditions for each of the target and reference genes. A standard curve derived from serial dilutions of a mixture of all samples was included in each run. The amplification conditions consisted of $10 \mathrm{~min}$ at $95^{\circ} \mathrm{C}, 50$ cycles of $10 \mathrm{~s}$ at $95^{\circ} \mathrm{C}$ and $15 \mathrm{~s}$ at $60^{\circ} \mathrm{C}$. Reaction samples had a final volume of $20 \mu \mathrm{L}$ consisting of LightCycler480 Probes Master (Roche), the specific primer/fluorogenic probe mix (Applied
Biosystems) and $5 \mu \mathrm{L}$ cDNA. Amplifications were performed using a LightCycler480 detection system (Roche). Data were processed using the standard curve method. Expression of target genes was corrected by a normalisation factor that was calculated based on the expression of the three reference genes $(G A P D H$, HPRT-1 and PPIA).

\section{Sputum induction, processing and analysis of IL-1 $\alpha$ and IL- $1 \beta$ by ELISA}

Sputum induction and processing was performed as described previously [20]. Briefly, subjects inhaled sterile, pyrogen-free, hypertonic, nebulised saline at increasing concentrations of $\mathrm{NaCl}$ $(3,4$ and $5 \%)$ over a 5 -min period after inhalation of salbutamol $(2 \times 200 \mu \mathrm{g})$. Subsequently, subjects were encouraged to cough and expectorate an adequate sample. Sputum plugs were selected, transferred to a polystyrene tube and mixed with dithiothreitol (DTT) (10\% Sputalysin; Boehringer-Calbiochem Corp, San Diego, CA, USA) (four times the weight of the sputum plugs) for $30 \mathrm{~s}$ by vortex and $15 \mathrm{~min}$ by tube rocker. Next, an amount of PBS equal to the volume of DTT was added. The sample was incubated for $5 \mathrm{~min}$, filtered and centrifuged for separation into cell and cell-free supernatant fractions. The supernatants was aspirated, aliquoted and stored at $-80^{\circ} \mathrm{C}$ until further analysis. The cell pellet was processed as previously described [20] and subjected to differential cell counts.

We determined IL- $1 \alpha$ and IL- $1 \beta$ in sputum supernatants using commercially available ELISA kits (R\&D Systems). Sputum supernatants were diluted four-fold and analysed according to the manufacturer's instructions.

\section{Statistical analysis}

Statistical analysis was performed with SigmaStat software (version 15.0; SPSS Inc., Chicago, IL, USA) using nonparametric tests (Kruskal-Wallis test and Mann-Whitney U-test) for variables without normal distribution, parametric tests (ANOVA and unpaired t-test) for variables with normal distribution and Fisher's exact test. Data are presented as mean \pm SEM. A $p$-value of $<0.05$ was considered significant.

\section{RESULTS}

\section{IL-1RI is critically implicated in subacute CS-induced pulmonary inflammation}

To elucidate whether the pulmonary inflammation upon 4 weeks (subacute) CS exposure is IL-1RI dependent, we evaluated inflammatory cells and cytokines in BAL fluid and lung tissue of WT and IL-1RI KO mice. In WT mice, exposure to CS significantly increased the total number of BAL cells, and absolute numbers of neutrophils, DCs, and CD4+ and CD8+ Tcells in BAL fluid (fig. 1). In contrast, IL-1RI KO mice were significantly protected against CS-induced accumulation of inflammatory cells in BAL fluid (fig. 1). Also, in lung digests, CS-induced accumulation of macrophages, neutrophils, DCs, and activated (CD69+) CD4+ and activated CD8+ T-cells was significantly impaired in IL-1RI KO mice, compared with WT mice (fig. 2).

Levels of the pro-inflammatory IL-1 family members IL-1 $\alpha$ and IL-1 $\beta$ were significantly upregulated in lungs of WT mice upon CS exposure, as measured by ELISA in lung homogenates (fig. 3). In IL-1RI KO mice, the CS-induced increase of IL- $1 \alpha$ 

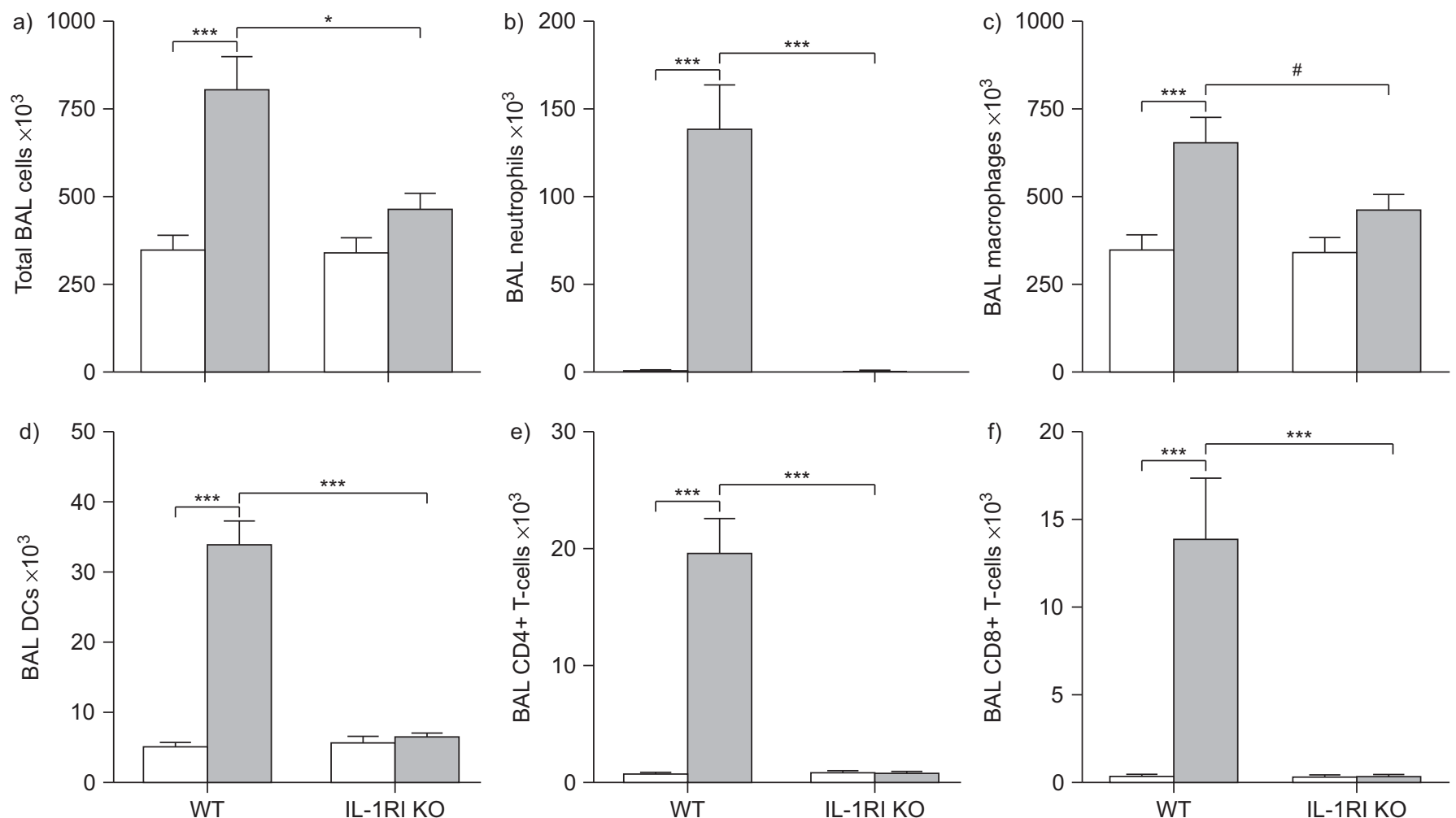

FIGURE 1. Effect of cigarette smoke (CS) exposure and interleukin-1 receptor (IL-1R)I deficiency on the total number of bronchoalveolar lavage (BAL) cells and cell subsets in BAL fluid. BAL cell counts in wild-type (WT) and IL-1RI knockout (KO) mice upon 4 weeks of exposure to air ( $\square$ ) or CS ( $\square$ ): a) total BAL cells, b) neutrophils, c) macrophages, d) dendritic cells (DCs), e) CD4+ T-cells and f) CD8+ T-cells. All cell types were counted by flow cytometry, except for the neutrophils, which were determined by cytospin counts. Data are presented as mean \pm SEM $\left(n=10\right.$ animals per group). ${ }^{*}: p=0.063 ; *^{*} p<0.05 ;{ }^{* *} p<0.001$.
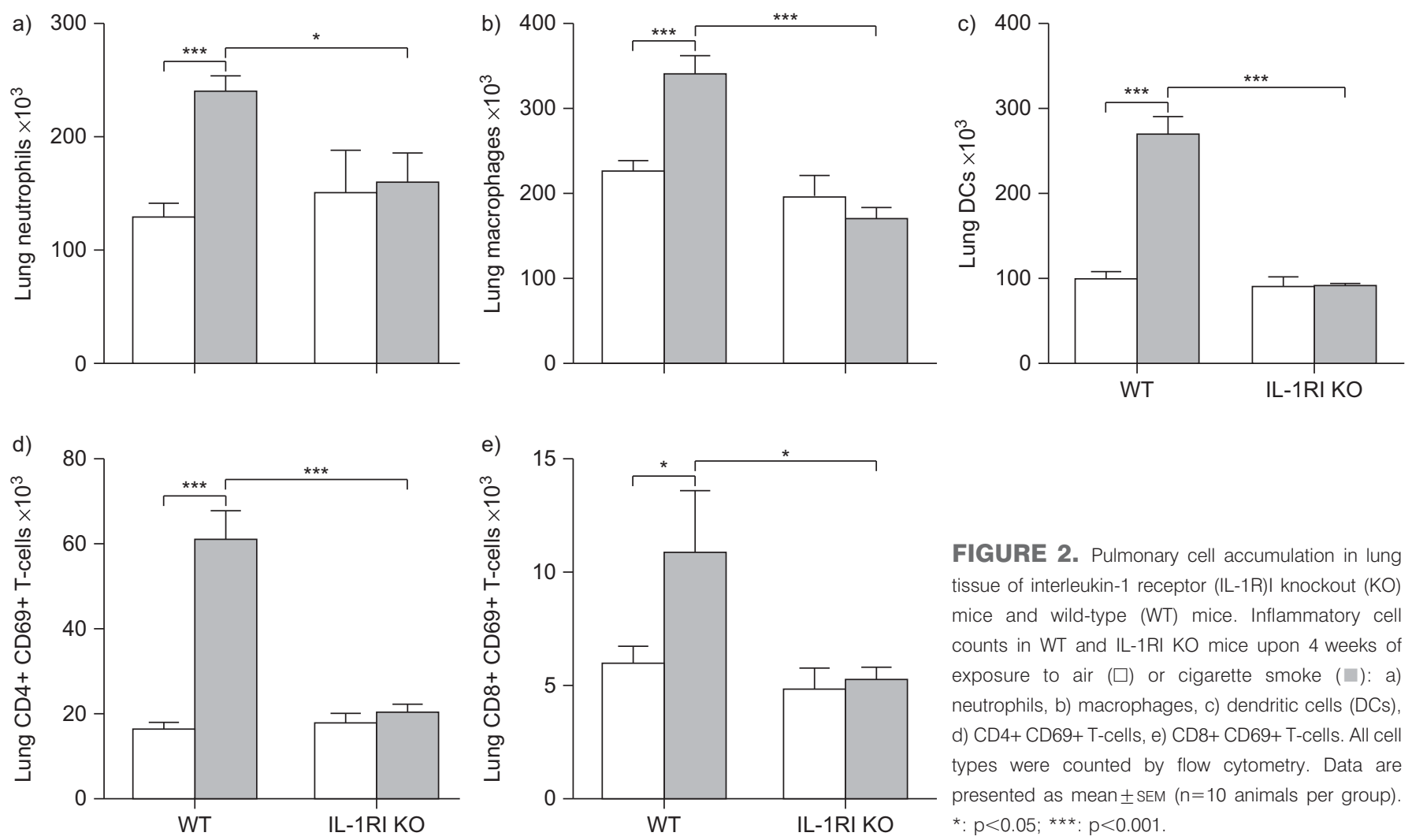

FIGURE 2. Pulmonary cell accumulation in lung tissue of interleukin-1 receptor (IL-1R)I knockout (KO) mice and wild-type (WT) mice. Inflammatory cell counts in WT and IL-1RI KO mice upon 4 weeks of exposure to air $(\square)$ or cigarette smoke ( $\square$ ): a) neutrophils, b) macrophages, c) dendritic cells (DCs), d) CD4+ CD69+ T-cells, e) CD8+ CD69+ T-cells. All cell types were counted by flow cytometry. Data are presented as mean \pm SEM $(n=10$ animals per group). $*: p<0.05 ; * * *: p<0.001$. 

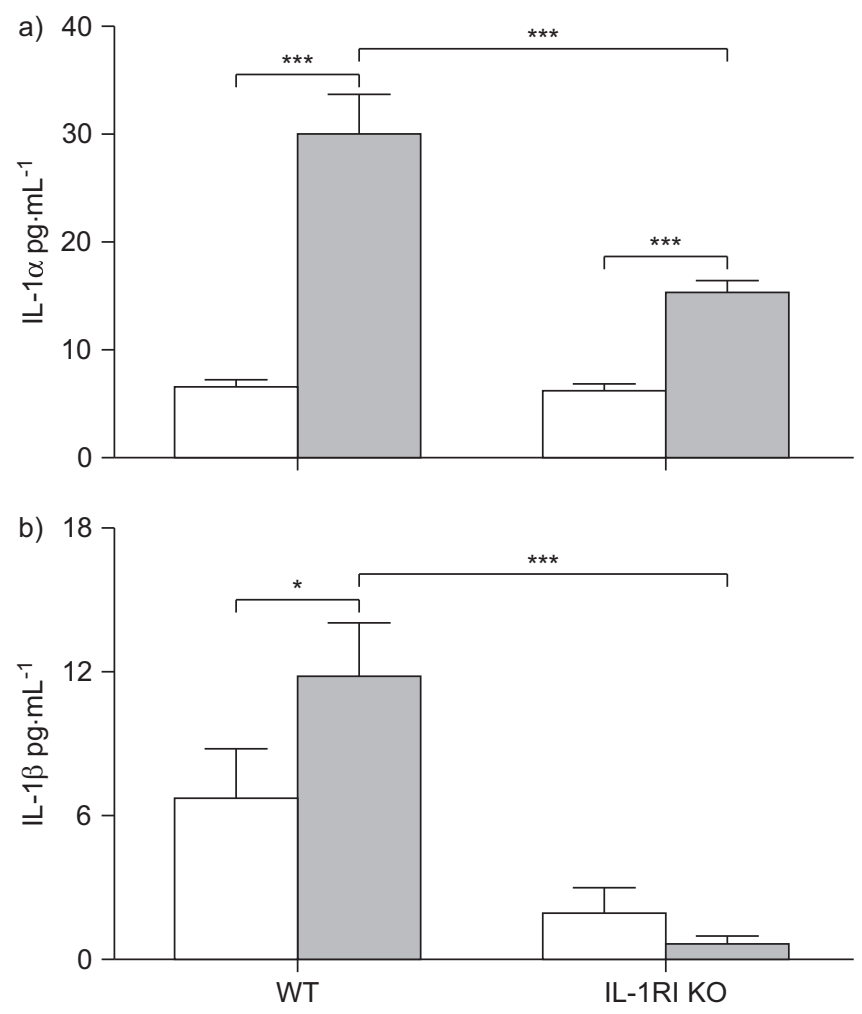

FIGURE 3. Effect of cigarette smoke (CS) exposure and interleukin-1 receptor (IL-1R)I deficiency on pulmonary levels of interleukin (IL)- $1 \alpha$ and IL-1 $\beta$. a) IL-1 $\alpha$ and b) IL-1 $\beta$ protein levels in lung homogenates of wild-type (WT) and IL-1RI knockout (KO) mice upon 4 weeks of exposure to air $(\square)$ or CS $(\square)$, as measured by ELISA. Data are presented as mean \pm SEM ( $n=10$ animals per group). ${ }^{*}: p<0.05$; $* * *: p<0.001$.

and IL-1 $\beta$ was significantly attenuated, compared with WT mice (fig. 3).

\section{CS-induced pulmonary inflammation is attenuated upon IL- $1 \alpha$ or IL-1 $\beta$ neutralisation}

To elucidate whether IL- $1 \alpha$ or IL- $1 \beta$ is the predominant ligand for IL-1RI in CS-induced responses, we exposed C57BL/ 6 mice to CS for 3 days (acute exposure) and injected them i.v. on days 1 and 3 with hamster IgG (isotype control) or neutralising antibodies against IL- $1 \alpha$ or IL-1 $\beta$. Acute exposure to CS resulted in a significant increase in BAL neutrophils in isotype control-treated mice (fig. 4). In contrast, mice treated with antiIL- $1 \alpha$ or anti-IL-1 $\beta$ antibodies were significantly protected against the CS-induced increase in total BAL cells and in BAL neutrophils (fig. 4). BAL macrophages and CD4+ and CD8+ Tcells were not (within the time-course of the experiment) significantly increased upon acute CS exposure in either isotype control or neutralising antibody-treated groups (data not shown).

\section{CS-induced pulmonary inflammation is independent of the NIrp3 inflammasome}

To investigate whether the Nlrp3/caspase-1/IL-1 $\beta$ axis is involved in CS-induced responses, we first evaluated pulmonary inflammation upon subacute CS exposure in Nlrp3 KO versus WT mice. The accumulation of macrophages, neutrophils,

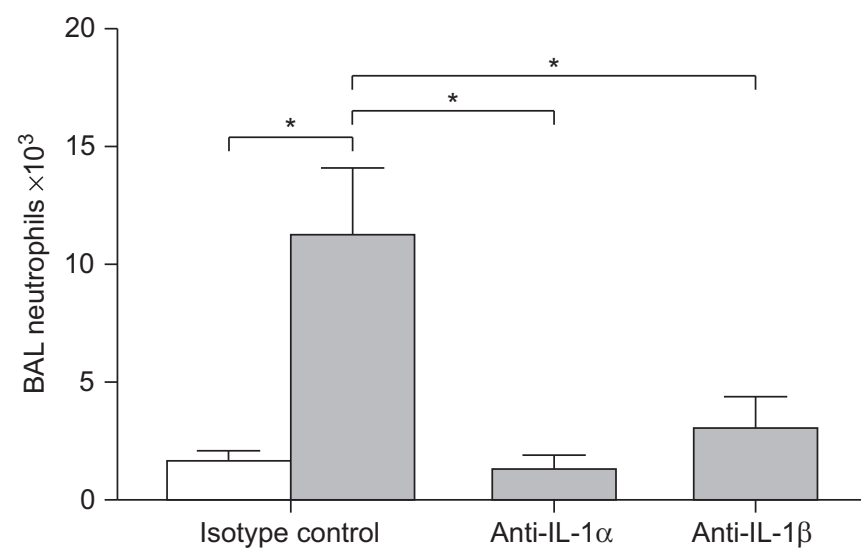

FIGURE 4. Effect of cigarette smoke (CS) exposure and interleukin (IL)-1 $\alpha$ or IL-1 $\beta$ neutralisation on the total number of neutrophils in bronchoalveolar lavage (BAL) fluid. BAL neutrophils in wild-type mice upon 3 days of exposure to air ( $\square$ ) or CS (匹), injected intravenously on days 1 and 3 with anti-IL-1 $\alpha$, anti-IL-1 $\beta$ or hamster immunoglobulin $G$ isotype control antibodies. Neutrophils were determined by cytospin counts. Data are presented as mean $\pm \operatorname{SEM}(n=8$ animals per group). $*$ : $p<0.05$

DCs and CD4+ and CD8+ T-cells in BAL fluid upon 4 weeks of CS exposure was similar between WT and Nlrp3 KO mice (fig. 5), indicating that the Nlrp3 inflammasome is not critical in subacute CS-induced pulmonary inflammation. Since the Nlrp3 inflammasome is, in contrast to other inflammasomes [8], described as playing an important role in acute responses, we also studied the involvement of Nlrp3 in acute (3 days) CS exposure. We observed that the Nlrp3 deficiency did not affect acute pulmonary inflammation, since numbers of macrophages, neutrophils, DCs and CD4+ and CD8+ T-cells were not significantly different between WT and Nlrp3 KO mice (data not shown).

\section{CS-induced pulmonary inflammation is not affected by caspase-1 deficiency}

Since pro-caspase- 1 can be activated by mechanisms other than the Nlrp3 inflammasome [7], we investigated the role of caspase-1 deficiency in CS-induced pulmonary inflammation. Caspase- $1 \mathrm{KO}$ mice were not protected against subacute CSinduced pulmonary accumulation of macrophages, neutrophils, DCs and CD4+ and CD8+ T-cells in BAL fluid (fig. 6), indicating that caspase- 1 is not critically implicated in CSinduced inflammatory responses.

\section{Expression levels of IL-1 $\alpha$ and IL-1ß in CS-exposed NIrp3 and caspase-1 KO mice}

We determined IL- $1 \alpha$ and IL- $1 \beta$ protein levels upon CS exposure (in lung homogenates and BAL fluid), because they both exert their biological responses by binding to IL-1RI. IL-1 $\alpha$ levels were increased in both BAL fluid and lung homogenates of WT mice (fig. 7a and b). Importantly, the CS-induced increase in IL-1 $\alpha$ was not different between WT, Nlrp3 KO and caspase-1 $\mathrm{KO}$ mice (fig. $7 \mathrm{a}$ and $\mathrm{b}$ ). IL-1 $\beta$ levels in BAL fluid were below the detection limit of the ELISA assay in both air- and CS-exposed groups. In lung homogenate, levels of IL-1 $\beta$ were affected by both Nlrp3 and caspase-1 deficiency, since CS-exposed Nlrp3 $\mathrm{KO}$ and caspase-1 KO mice had significantly lower levels of 

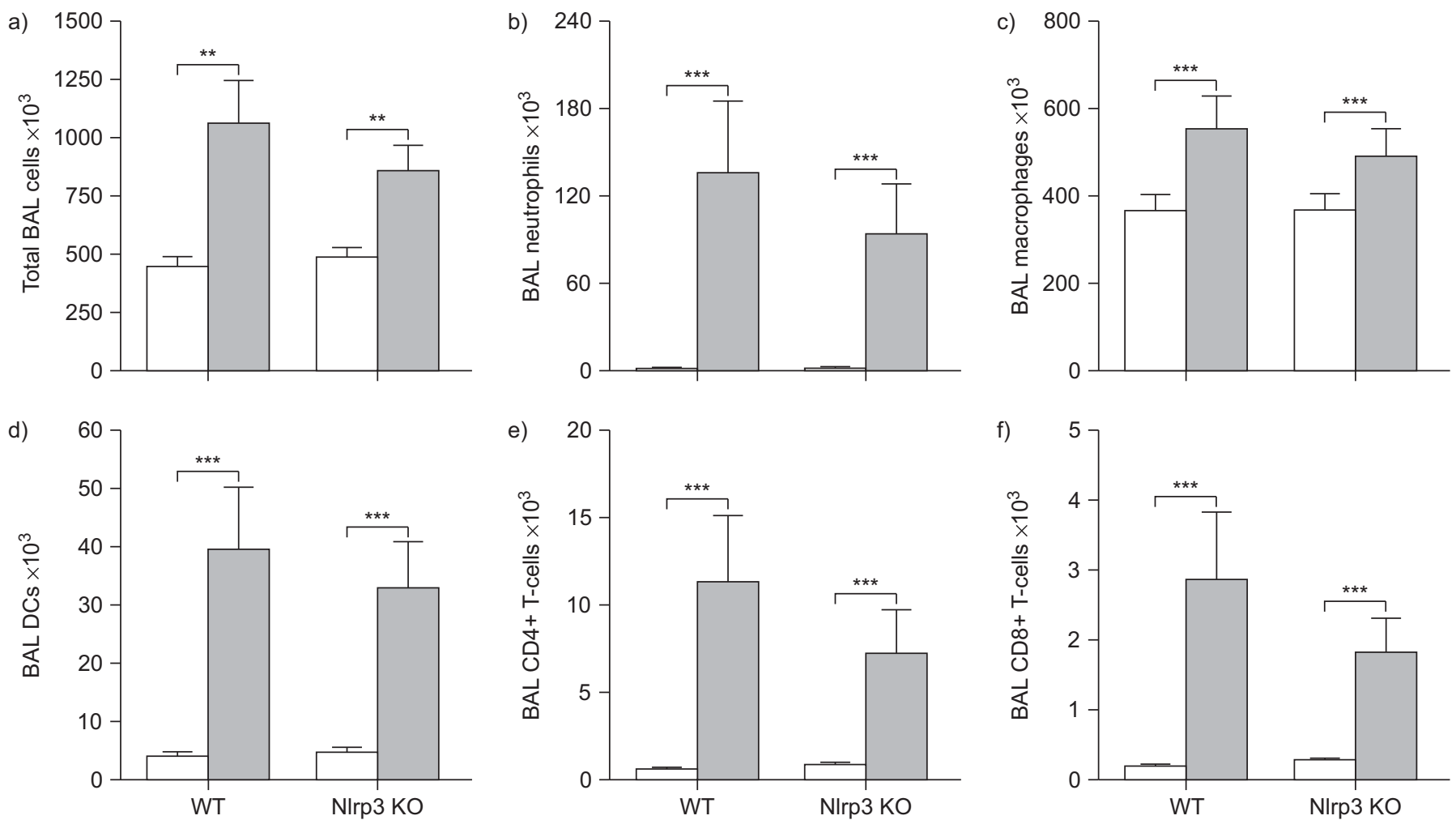

FIGURE 5. Effect of cigarette smoke (CS) exposure and NIrp3 deficiency on the total number of bronchoalveolar lavage (BAL) cells and cell subsets in BAL fluid. BAL cell counts in wild-type (WT) and NIrp3 knockout (KO) mice upon 4 weeks of exposure to air ( $\square$ ) or CS ( $\square$ ): a) total BAL cells, b) neutrophils, c) macrophages, d) dendritic cells (DCs), e) CD4+ T-cells and f) CD8+ T-cells. All cell types were counted by flow cytometry, except for the neutrophils, which were determined by cytospin counts. Data are presented as mean \pm SEM $\left(n=10\right.$ animals per group). ${ }^{* *}: p<0.01 ; * * *: p<0.001$

IL-1 $\beta$, compared with CS-exposed WT mice (fig. 7c and d). Since both inflammatory cell recruitment and IL- $1 \alpha$ levels are not impaired in CS-exposed Nlrp3 and caspase-1 KO mice, while the levels of IL-1 $\beta$ in these mice are significantly attenuated, IL$1 \alpha$ may be an important trigger for IL-1RI in CS-induced responses.

\section{IL-1 $\alpha$ mRNA and IL-1 $\beta$ protein levels are increased in patients with COPD}

To evaluate mRNA expression of IL- $1 \alpha$ and IL-1 $\beta$ by quantitative RT-PCR, we extracted mRNA from total lung tissue of a study population containing never-smokers, smokers without COPD and patients with COPD. The demographic, clinical and lung function characteristics of the study subjects are presented in table 1 . Levels of IL- $1 \alpha$ mRNA were significantly and by at least two-fold increased in both smokers without COPD and patients with COPD, compared with never-smokers (fig. 8a). IL-1 $\beta$ mRNA expression was also increased in smokers with and without COPD, but this increase did not reach statistical significance (fig. 8b).

In addition, protein levels of IL- $1 \alpha$ and IL-1 $\beta$ in induced sputum of never-smokers, smokers without COPD and patients with COPD were determined by ELISA. The characteristics of the study subjects who underwent sputum induction are listed in table 2 . In accordance with the mRNA levels, protein levels of IL-1 $\alpha$ increased in the induced sputum of smokers and patients with COPD compared with never-smokers, but this did not reach statistical significance (fig. 8c). However, protein levels of IL-1 $\beta$ were significantly increased in patients with COPD compared with never-smokers and smokers without airflow limitation (fig. 8d).

\section{DISCUSSION}

Using a mouse model of CS exposure, we have reinforced previous findings that CS-induced pulmonary inflammatory cell and cytokine accumulation is IL-1RI-dependent. Importantly, our in vivo study in mice is the first to report that the impaired inflammatory response in CS-exposed IL-1RI KO mice can not only be mimicked by neutralisation of IL-1 $\beta$, but also with anti-IL-1 $\alpha$ antibodies. Interestingly, CS-induced pulmonary inflammation in mice is not critically mediated by the Nlrp3/caspase-1/IL-1 $\beta$ axis, again suggesting a role for IL- $1 \alpha$ and/or alternative activation of IL-1 $\beta$. Finally, using a translational approach, we demonstrated increased levels of IL- $1 \alpha$ and IL- $1 \beta$ in lungs and sputum of patients with COPD, respectively, compared with never-smokers.

We have shown that IL-1RI KO mice are protected against pulmonary inflammation induced by subacute (4 weeks) CS exposure, which is in accordance with previous reports about IL-1RI KO mice exposed to acute or chronic CS [12, 13]. However, our study is the first to report a protection of IL-1RI KO mice against CS-induced accumulation of DCs and CD4+ and CD8+ T-cells. Our study hereby reinforces the important role for IL-1RI in CS-induced inflammatory responses. 

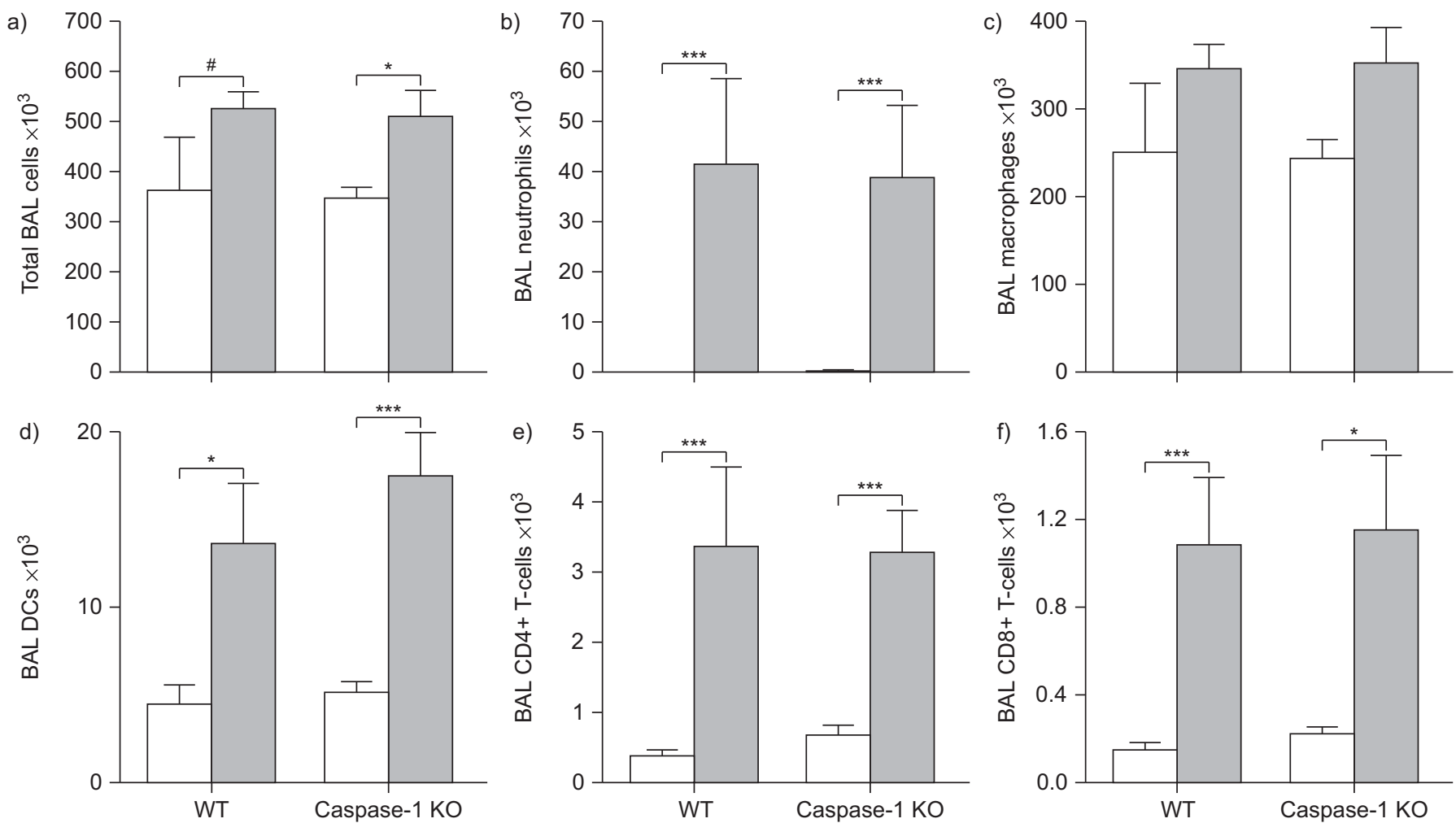

FIGURE 6. Effect of cigarette smoke (CS) exposure and caspase-1 deficiency on the total number of bronchoalveolar lavage (BAL) cells and cell subsets in BAL fluid. BAL cell counts in wild-type (WT) and caspase-1 knockout (KO) mice upon 4 weeks of exposure to air ( $\square$ ) or CS ( $\square$ ): a) total BAL cells, b) neutrophils, c) macrophages, d) dendritic cells (DCs), and e) CD4+ T-cells and f) CD8+ T-cells. All cell types were counted by flow cytometry, except for the neutrophils, which were determined by cytospin counts. Data are presented as mean \pm SEM ( $n=8$ animals per group). ${ }^{*}: p=0.073 ;{ }^{*} p<0.05 ;{ }^{* * *} p<0.001$

However, it was still unclear which pro-inflammatory ligand, IL- $1 \alpha$ or IL- $1 \beta$, predominantly stimulates the common receptor upon CS exposure.

We demonstrated that mice treated with anti-IL- $1 \alpha$ or anti-IL$1 \beta$ antibodies were significantly protected against acute CSinduced increases in BAL neutrophils, suggesting a role for both IL- $1 \alpha$ and IL- $1 \beta$ in CS-induced inflammation. While the observed protective effect of anti-IL-1 $\beta$ confirms the results of CASTRO et al. [21], this is, to our knowledge, the first report of an attenuated CS-induced inflammation by neutralisation of IL- $1 \alpha$. The involvement of IL- $1 \alpha$ in CS-induced inflammatory responses is underappreciated, although IL- $1 \alpha$ has been described to play an important role in inflammation (e.g. mice deficient in IL-1 $\alpha$ are resistant to experimental colitis [22]). Also, in sterile inflammation, IL- $1 \alpha$, but not IL-1 $\beta$, drives the neutrophilic inflammatory response to cell injury [23]. Multiple in vivo studies in mice describe the levels of IL-1 $\beta$ upon CS, but these levels seem to depend strongly on the mouse strain, the specimen tested (BAL fluid or lung homogenate) and the exposure time $[12,13,21,24]$. In general, IL-1 $\beta$ has been described to be upregulated and implicated in early responses upon CS exposure [12, 21], but not chronic CS exposure [13].

We next studied the role of IL-1 $\beta$ activation by the Nlrp3/ caspase-1 pathway in CS-induced inflammation. We found that accumulation of inflammatory cells in the airways and lungs upon subacute CS exposure is independent of Nlrp3 and caspase-1. In contrast, CHURG et al. [13] previously demonstrated, in an acute CS model, that administration of a selective caspase-1 inhibitor resulted in diminished CS-induced pulmonary inflammation. However, their experiments with caspase-1 inhibition are all in an acute setting, which is different from our prolonged CS exposure. The processes driving pulmonary inflammation upon CS exposure probably change over time, with different pathways implicated in the initiation and persistence of inflammatory responses. Similarly, the role for caspase- 1 in experimental arthritis also depends on the phase of the disease (acute or chronic), as described by JOOSTEN et al. [25]. Since COPD is a chronic inflammatory disease, animal models that mimic and unravel the molecular mechanisms of responses to prolonged CS exposure are of great value and interest. Interestingly, we have found that the pulmonary cell accumulation in CS-exposed Nlrp3 KO and caspase-1 KO mice is not impaired, although they have lower pulmonary levels of IL-1 $\beta$ upon CS exposure. This again suggests an important role for IL- $1 \alpha$, the other proinflammatory ligand of IL-1RI, which is not impaired in Nlrp3 $\mathrm{KO}$ and caspase-1 $\mathrm{KO}$ mice upon prolonged exposure to $\mathrm{CS}$.

To support our findings in the mouse COPD model, we performed a translational study and investigated IL- $1 \alpha$ and IL$1 \beta$ in never-smokers, smokers without COPD and patients with COPD. We found that IL- $1 \alpha$ mRNA expression was significantly increased in total lung tissue of smokers without airflow limitation and patients with COPD, compared with never-smokers. Although we observed the same trends for the IL-1 $\alpha$ protein levels in induced sputum, these differences did 

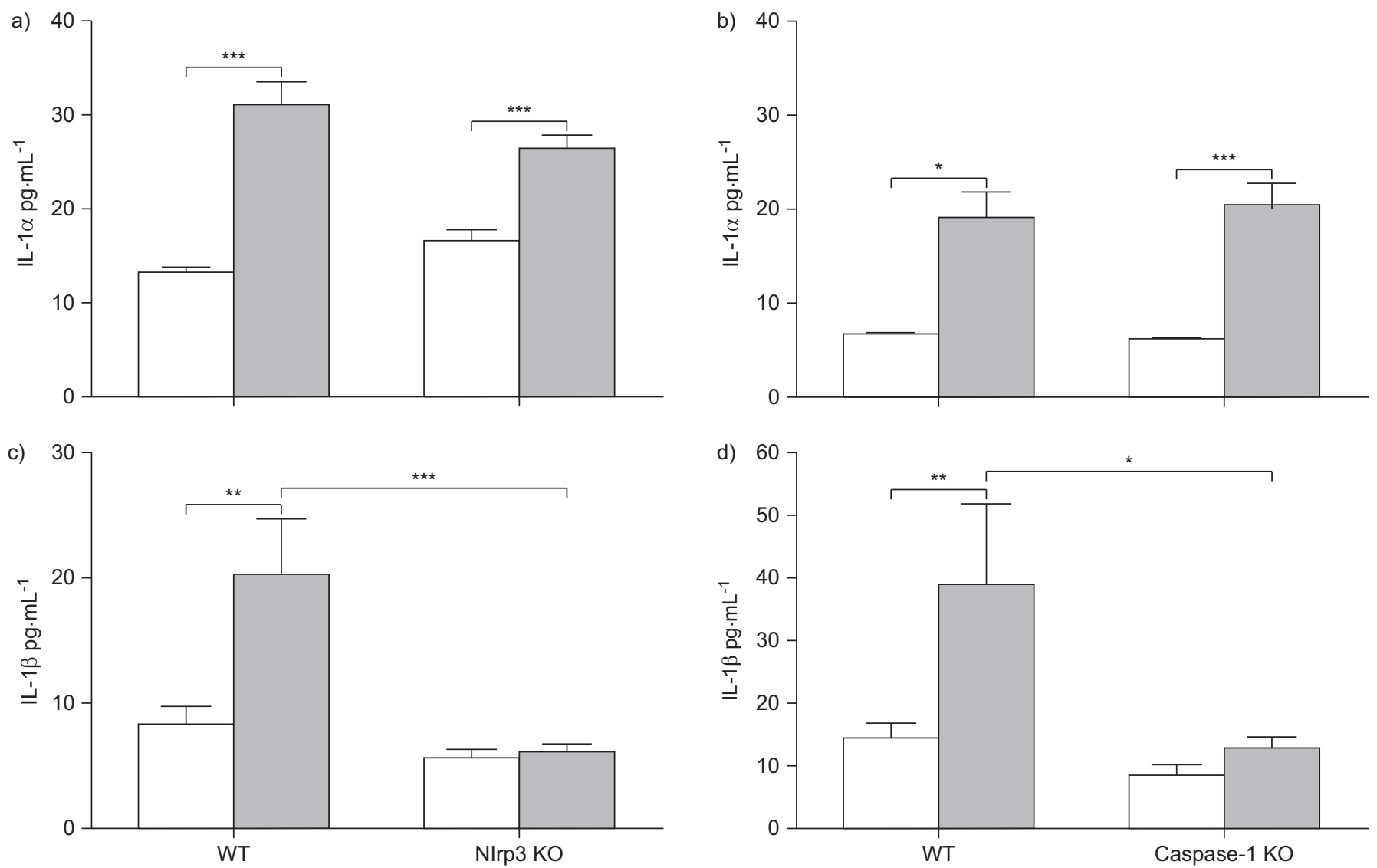

FIGURE 7. Effect of cigarette smoke (CS) exposure, Nlrp3 deficiency and caspase-1 deficiency on pulmonary levels of interleukin (IL)- $1 \alpha$ and IL-1 $\beta$. IL-1 $\alpha$ levels in lung homogenates of a) Nlrp3 knockout (KO) and b) caspase-1 KO mice upon 4 weeks of exposure to air ( $\square$ ) or CS ( $\square$ ), as measured by ELISA. IL-1 $\beta$ levels in lung homogenates of $\mathrm{c}$ ) NIrp3 $\mathrm{KO}$ and d) caspase- $1 \mathrm{KO}$ mice upon 4 weeks of exposure to air or CS, as measured by ELISA. Data are presented as mean \pm SEM ( $\mathrm{n}=8-10$ animals per group). *: $p<0.05 ;{ }^{* *} p<0.01 ;{ }^{* *} p<0.001$.

\begin{tabular}{|c|c|c|c|c|}
\hline \multirow[t]{2}{*}{ TABLE 1} & \multicolumn{4}{|c|}{$\begin{array}{l}\text { Characteristics of study subjects for lung mRNA } \\
\text { analysis by quantitative RT-PCR }\end{array}$} \\
\hline & & $\begin{array}{l}\text { Never- } \\
\text { smokers }\end{array}$ & Smokers & COPD \\
\hline \multicolumn{2}{|l|}{ Subjects } & 10 & 18 & 29 \\
\hline \multicolumn{2}{|c|}{ Males/females } & $3 / 7^{\star \star \star}$ & $12 / 6^{\star \star *}$ & $26 / 3^{* \star *}$ \\
\hline \multicolumn{2}{|l|}{ Age yrs } & $61(50-70)$ & $61(52-69)$ & $67(59-72)$ \\
\hline \multicolumn{2}{|c|}{ Current-smokers/ex-smokers } & & $7 / 11$ & $17 / 12$ \\
\hline \multicolumn{2}{|c|}{ Smoking history pack-yrs } & $0(0-0)$ & $35(19-46)^{\star}$ & $40(30-55)^{\star}$ \\
\hline \multicolumn{2}{|c|}{ Post-BD FEV 1 L } & $2.6(2.0-3.0)$ & $3.2(2.7-3.5)$ & $2(1.8-2.6)^{\#}$ \\
\hline \multicolumn{2}{|c|}{ Post-BD FEV 1 \% pred } & $103(84-104)$ & 108 (93-113) & $66(55-77)^{\star, \#}$ \\
\hline \multicolumn{2}{|c|}{ Post-BD FEV $1 /$ FVC \% } & $77(73-84)$ & $77(71-81)$ & $56(51-61)^{\star, \#}$ \\
\hline \multicolumn{2}{|l|}{ ICS yes/no } & $0 / 10^{* \star *}$ & $0 / 18^{\star \star \star}$ & $11 / 18^{* * *}$ \\
\hline
\end{tabular}

Data are presented as $\mathrm{n}$ or median (interquartile range). COPD: chronic obstructive pulmonary disease; BD: bronchodilator; FEV1: forced expiratory volume in $1 \mathrm{~s}$; \% pred: \% predicted; FVC: forced vital capacity; ICS: inhaled corticosteroid. *: $p<0.05$ versus never-smokers by Mann-Whitney U-test; \#: $p<0.05$ versus smokers by Mann-Whitney U-test; ${ }^{* \star *}: p<0.001$ by Fisher's exact test. not reach statistical significance. This may be due to the great heterogeneity of COPD, but may also be explained by the origin of the samples and the different sources of IL- $1 \alpha$ and IL$1 \beta$ [26]. As IL-1 $\alpha$ is released as a danger signal by necrotic cells, induced sputum may not be the ideal compartment in which to measure IL- $1 \alpha$ levels, especially since the viability of the cells in our sputum inductions was $\sim 90 \%$. In contrast, IL- $1 \beta$ is mainly released by activated macrophages. Therefore, we found significantly increased protein levels of IL-1 $\beta$ in the induced sputum of patients with COPD, compared with neversmokers and smokers without airflow limitation. Although IL$1 \beta$ expression is well-studied in patients with COPD [27, 28], the major advantage of this study is that both IL-1RI ligands, IL- $1 \alpha$ and IL- $1 \beta$, were evaluated in the same subjects. Taken together with the in vivo findings in the mouse model, these data further suggest an important role for both IL- $1 \alpha$ and IL-1 $\beta$ in IL-1RI-driven inflammation in COPD.

Our findings could have important implications for the study of anti-inflammatory therapies for smoking-related diseases like COPD. Our data indicate that potential therapies targeting Nlrp3 or caspase- 1 might have limited benefit, compared with the efficacy of IL- $1 \alpha / \beta$ or IL-1RI antagonism. Recombinant human IL-1R antagonist (anakinra; Kineret ${ }^{\circledR}$, Biovitrium, Stockholm, Sweden) is already marketed for the treatment of 

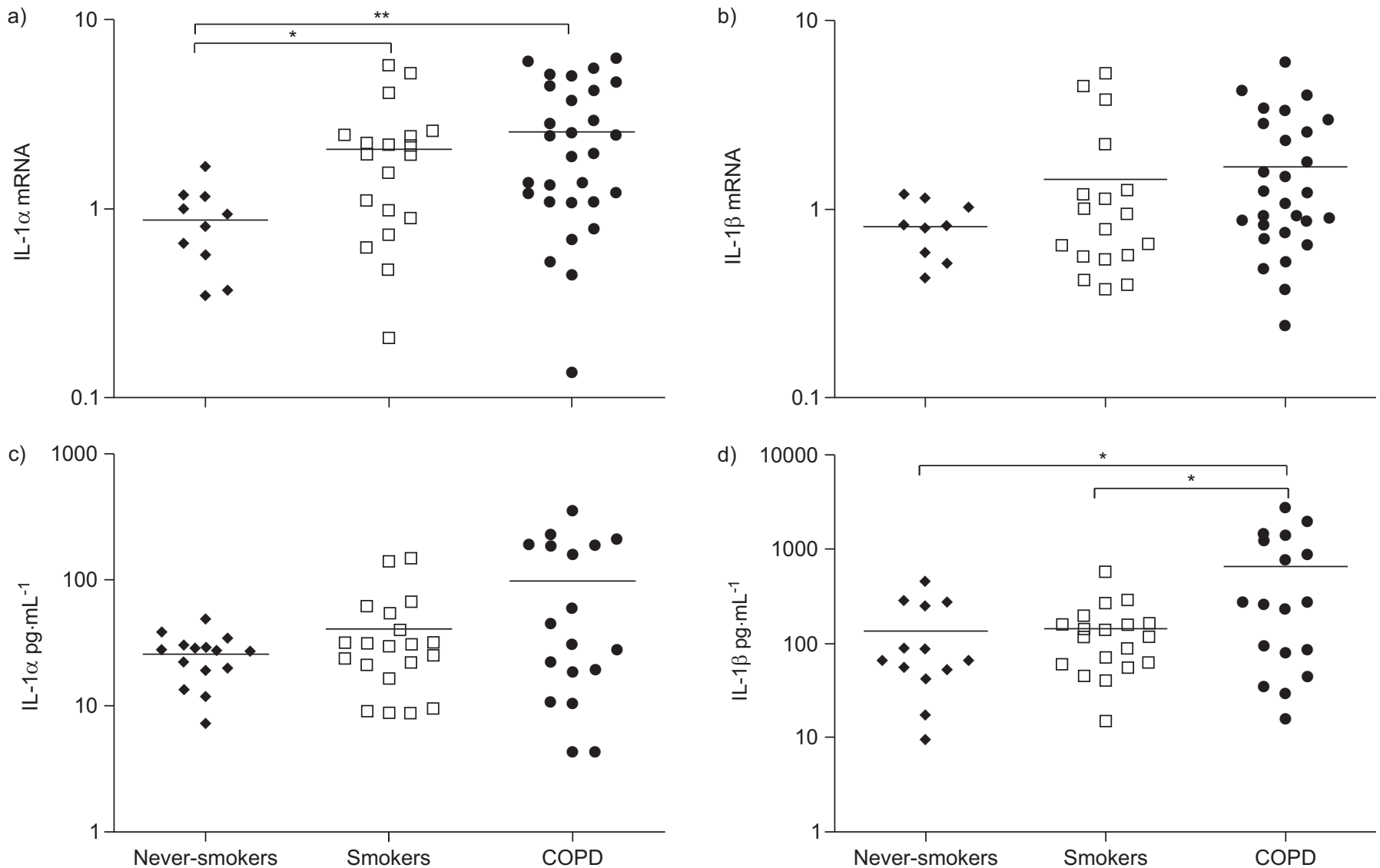

FIGURE 8. Expression of interleukin (IL)-1 receptor I ligands, IL-1 $\alpha$ and IL-1 $\beta$, in human subjects: never-smokers, smokers (without chronic obstructive pulmonary disease (COPD)) and patients with COPD. mRNA levels of a) IL-1 $\alpha$ and b) IL-1 $\beta$ in lung tissue of never-smokers $(n=10)$, smokers ( $n=18$ ) and patients with COPD ( $n=29)$, as measured by quantitative RT-PCR. mRNA levels were corrected using a calculated normalisation factor based on the mRNA expression of three reference genes (GAPDH, PPIA and HPRT-1). Protein levels of $c)$ IL-1 $\alpha$ and d) IL-1 $\beta$ in induced sputum of never-smokers $(n=15)$, smokers $(n=20)$ and patients with $\operatorname{COPD}(n=18) .{ }^{*}: p<0.05 ;{ }^{* *}: p<0.01$.

\section{TABLE 2 Characteristics of study subjects for sputum analysis by ELISA}

\begin{tabular}{|c|c|c|c|}
\hline Subjects & 15 & 20 & 18 \\
\hline Age yrs & $52(40-55)$ & $52(46-62)$ & $60(56-71)^{*, \#}$ \\
\hline Current-smokers/ex-smokers & & $13 / 7$ & $11 / 7$ \\
\hline Smoking history pack-yrs & $0.0(0.0-0.0)$ & $30(10-48)^{*}$ & $50(39-84)^{*, \#}$ \\
\hline Post-BD FEV $1 /$ FVC \% & $80(77-87)$ & 79 (76-83) & $58(49-66)^{*, \#}$ \\
\hline ICS yes/no & $0 / 15^{\star \star \star}$ & $0 / 20^{\star \star \star}$ & $8 / 10^{\star \star *}$ \\
\hline \multicolumn{4}{|l|}{ Sputum differential cell count $\%$} \\
\hline Macrophages & 57 (39-68) & $40(25-68)$ & $29(15-39)^{*, \#}$ \\
\hline Neutrophils & $42(28-59)$ & $58(30-73)$ & $65(54-81)^{*}$ \\
\hline Viability & 88 (79-92) & $90(85-95)$ & 89 (81-92) \\
\hline
\end{tabular}


rheumatoid arthritis, but it has, to our knowledge, not yet been examined clinically in patients with COPD.

In conclusion, we confirm that pulmonary inflammation upon CS exposure in mice is IL-1RI dependent, and demonstrate for the first time a crucial role for both IL- $1 \alpha$ and IL-1 $\beta$. Moreover, we have shown that pulmonary inflammation upon CS exposure is independent from IL-1 $\beta$ activation by the Nlrp3/ caspase-1 axis. Finally, we demonstrated increased levels of both IL- $1 \alpha$ and IL-1 $\beta$ in patients with COPD. These results suggest that not only IL- $1 \beta$ but also IL- $1 \alpha$ should be considered as important mediators in CS-induced inflammation and COPD.

\section{SUPPORT STATEMENT}

The research described in this article was supported by the Concerted Research Action of the University of Ghent (BOF/GOA; grants 01251504 and 01601009) and the Fund for Scientific Research Flanders (FWO Vlaanderen; grants G.0195.09 and G.0194.10). K.R. Bracke and T. Vanden Berghe are postdoctoral researchers funded by FWO Vlaanderen, and G.R. Van Pottelberge is a doctoral researcher funded by FWO Vlaanderen.

\section{STATEMENT OF INTEREST}

None declared.

\section{ACKNOWLEDGEMENTS}

The authors would like to thank G. Barbier, E. Castrique, I. De Borle, P. De Gryze, K. De Saedeleer, A. Goethals, M-R. Mouton, A. Neesen, C. Snauwaert, E. Spruyt and S. Verschraeghen (Laboratory for Translational Research in Obstructive Pulmonary Diseases, Dept of Respiratory Medicine, Ghent University Hospital, Ghent, Belgium) for their technical assistance. Nlrp3 $\mathrm{KO}$ mice were kindly provided by J. Tschopp (University of Lausanne, Lausanne, Switzerland) and housed in the animal facility at the Faculty of Medicine and Health Sciences (Ghent University).

\section{REFERENCES}

1 Buist AS, McBurnie MA, Vollmer WM, et al. International variation in the prevalence of COPD (the BOLD Study): a population-based prevalence study. Lancet 2007; 370: 741-750.

2 Global Initiative for Chronic Obstructive Pulmonary Disease. Global Strategy for the Diagnosis, Management, and Prevention of Chronic Obstructive Pulmonary Disease. www.goldcopd.org/ guidelines-global-strategy-for-diagnosis-management.html Date last updated: 2010.

3 Dinarello CA. Immunological and inflammatory functions of the interleukin-1 family. Annu Rev Immunol 2009; 27: 519-550.

4 Sims JE, Smith DE. The IL-1 family: regulators of immunity. Nat Rev Immunol 2010; 10: 89-102.

5 Bracke KR, D'hulst AI, Maes T, et al. Cigarette smoke-induced pulmonary inflammation and emphysema are attenuated in CCR6-deficient mice. J Immunol 2006; 177: 4350-4359.

6 Martinon F, Mayor A, Tschopp J. The inflammasomes: guardians of the body. Annu Rev Immunol 2009; 27: 229-265.

7 Petrilli V, Dostert C, Muruve DA, et al. The inflammasome: a danger sensing complex triggering innate immunity. Curr Opin Immunol 2007; 19: 615-622.

8 Warren SE, Mao DP, Rodriguez AE, et al. Multiple Nod-like receptors activate caspase 1 during Listeria monocytogenes infection. J Immunol 2008; 180: 7558-7564.
9 Franchi L, Eigenbrod T, Munoz-Planillo R, et al. The inflammasome: a caspase-1-activation platform that regulates immune responses and disease pathogenesis. Nat Immunol 2009; 10: 241-247.

10 Eigenbrod T, Park JH, Harder J, et al. Cutting edge: critical role for mesothelial cells in necrosis-induced inflammation through the recognition of IL-1 $\alpha$ released from dying cells. J Immunol 2008; 181: 8194-8198.

11 Keller M, Ruegg A, Werner S, et al. Active caspase-1 is a regulator of unconventional protein secretion. Cell 2008; 132: 818-831.

12 Doz E, Noulin N, Boichot E, et al. Cigarette smoke-induced pulmonary inflammation is TLR4/MyD88 and IL-1R1/MyD88 signaling dependent. J Immunol 2008; 180: 1169-1178.

13 Churg A, Zhou S, Wang $X$, et al. The role of interleukin-1 $\beta$ in murine cigarette smoke-induced emphysema and small airway remodeling. Am J Respir Cell Mol Biol 2009; 40: 482-490.

14 D'hulst AI, Vermaelen KY, Brusselle GG, et al. Time course of cigarette smoke-induced pulmonary inflammation in mice. Eur Respir J 2005; 26: 204-213.

15 Bracke KR, D'hulst AI, Maes T, et al. Cigarette smoke-induced pulmonary inflammation, but not airway remodelling, is attenuated in chemokine receptor 5-deficient mice. Clin Exp Allergy 2007; 37: 1467-1479.

16 Demoor T, Bracke KR, Vermaelen KY, et al. CCR7 modulates pulmonary and lymph node inflammatory responses in cigarette smoke-exposed mice. J Immunol 2009; 183: 8186-8194.

17 Robays LJ, Maes T, Lebecque S, et al. Chemokine receptor CCR2 but not CCR5 or CCR6 mediates the increase in pulmonary dendritic cells during allergic airway inflammation. J Immunol 2007; 178: 5305-5311.

18 Vermaelen KY, Carro-Muino I, Lambrecht BN, et al. Specific migratory dendritic cells rapidly transport antigen from the airways to the thoracic lymph nodes. J Exp Med 2001; 193: 51-60.

19 Vermaelen K, Pauwels R. Accurate and simple discrimination of mouse pulmonary dendritic cell and macrophage populations by flow cytometry: methodology and new insights. Cytometry 2004; 61A: $170-177$.

20 Demedts IK, Morel-Montero A, Lebecque S, et al. Elevated MMP12 protein levels in induced sputum from patients with COPD. Thorax 2006; 61: 196-201.

21 Castro P, Legora-Machado A, Cardilo-Reis L, et al. Inhibition of interleukin-1 $\beta$ reduces mouse lung inflammation induced by exposure to cigarette smoke. Eur J Pharmacol 2004; 498: 279-286.

22 Kamari Y, Werman-Venkert R, Shaish A, et al. Differential role and tissue specificity of interleukin- $1 \alpha$ gene expression in atherogenesis and lipid metabolism. Atherosclerosis 2007; 195: 31-38.

23 Chen CJ, Kono H, Golenbock D, et al. Identification of a key pathway required for the sterile inflammatory response triggered by dying cells. Nat Med 2007; 13: 851-856.

24 Botelho FM, Gaschler GJ, Kianpour S, et al. Innate immune processes are sufficient for driving cigarette smoke-induced inflammation in mice. Am J Respir Cell Mol Biol 2010; 42: 394-403.

25 Joosten LA, Netea MG, Fantuzzi G, et al. Inflammatory arthritis in caspase 1 gene-deficient mice: contribution of proteinase 3 to caspase 1-independent production of bioactive interleukin-1 $\beta$. Arthritis Rheum 2009; 60: 3651-3662.

26 Chen GY, Nunez G. Sterile inflammation: sensing and reacting to damage. Nat Rev Immunol 2010; 10: 826-837.

27 Gessner C, Scheibe R, Wotzel M, et al. Exhaled breath condensate cytokine patterns in chronic obstructive pulmonary disease. Respir Med 2005; 99: 1229-1240.

28 Sapey E, Ahmad A, Bayley D, et al. Imbalances between interleukin-1 and tumour necrosis factor agonists and antagonists in stable COPD. J Clin Immunol 2009; 29: 508-516. 